\title{
PROGRESS CONCERNING THE LOCAL-GLOBAL PRINCIPLE FOR ZERO-CYCLES ON ALGEBRAIC VARIETIES
}

\author{
YONGQI LIANG
}

\begin{abstract}
We consider zero-cycles on algebraic varieties defined over number fields. The Hasse principle and weak approximation property are obstructed by the Brauer group of the variety and it is conjectured to be the only obstruction for all proper smooth varieties by Colliot-Thélène, Sansuc, Kato and Saito. We summarize the progress related to this conjecture, particularly for higher dimensional varieties such as fibrations and homogeneous varieties.
\end{abstract}

1. Introduction. Let $k$ be a number field, and let $\Omega$ be the set of places of $k$. In the present paper, the main consideration is the local-global principle for 0 -cycles on algebraic varieties $X$, which are proper smooth and geometrically integral over $k$. We simply write $X_{v}=X \otimes_{k} k_{v}$ for all $v \in \Omega$, and we denote the cohomological Brauer group of $X$ by

$$
\operatorname{Br}(X)=\mathrm{H}_{\text {ét }}^{2}\left(X, \mathbb{G}_{m}\right) .
$$

For any abelian group $M$, denote $\operatorname{Coker}(M \stackrel{n}{\rightarrow} M)$ by $M / n$, for any non zero integer $n$.

In the 1970's, Manin defined the following pairing between the local points of $X$ and the Brauer group of $X$, cf., [33].

2010 AMS Mathematics subject classification. Primary 11G35, 14C25, 14 G25.

Keywords and phrases. Brauer and Manin's obstruction, Hasse principle, weak approximation, zero-cycles.

This survey is based on a talk given by the author at the 6th Asian Mathematical Conference, Busan, Korea, June 30-July 4, 2013.

Received by the editors on September 17, 2013 and in revised form on September 3, 2014. 


$$
\begin{aligned}
\prod_{v \in \Omega} X\left(k_{v}\right) \times \operatorname{Br}(X) & \longrightarrow \mathbb{Q} / \mathbb{Z} \\
\left(\left\{x_{v}\right\}_{v \in \Omega}, b\right) & \longmapsto \sum_{v \in \Omega} \operatorname{inv}_{v}\left(b\left(x_{v}\right)\right),
\end{aligned}
$$

where $\operatorname{inv}_{v}: \operatorname{Br}\left(k_{v}\right) \rightarrow \mathbb{Q} / \mathbb{Z}$ is the local invariant. Denote the set of families of local points that are orthogonal to the subset $B$ of $\operatorname{Br}(X)$ by

$$
\left[\prod_{v \in \Omega} X\left(k_{v}\right)\right]^{B} .
$$

It is a closed subset of

$$
\prod_{v \in \Omega} X\left(k_{v}\right)
$$

by the continuity in the first component of the pairing. According to the global class field theory,

$$
\operatorname{Br}(k) \longrightarrow \bigoplus_{v \in \Omega} \operatorname{Br}\left(k_{v}\right) \longrightarrow \mathbb{Q} / \mathbb{Z}
$$

is a complex, the diagonal image of the set of rational points $X(k)$, as well as its closure, and is contained in the Brauer and Manin set

$$
\left[\prod_{v \in \Omega} X\left(k_{v}\right)\right]^{\operatorname{Br}(X)} .
$$

Hence, the Brauer group gives an obstruction to the Hasse principle and to the weak approximation for rational points.

Terminology. For a family of algebraic varieties, we say that the Brauer and Manin obstruction is the only obstruction to the Hasse principle for rational points if,

$$
\left[\prod_{v \in \Omega} X\left(k_{v}\right)\right]^{\operatorname{Br}(X)} \neq \emptyset
$$

implies that $X(k) \neq \emptyset$. 
We say that the Brauer and Manin obstruction is the only obstruction to weak approximation for rational points if $X(k)$ is dense in

$$
\left[\prod_{v \in \Omega} X\left(k_{v}\right)\right]^{\operatorname{Br}(X)}
$$

The Brauer and Manin obstruction explains the failure of the Hasse principle and weak approximation for rational points on several families of algebraic varieties. However, examples constructed by Skorobogatov [39], by Poonen [35], and by Harpaz and Skorobogatov [23], show that this is not always the case. All of these examples are at least dimension 2 , and they are not (geometrically) rationally connected varieties. It is believed that for smooth projective curves [38], and for rationally connected varieties [10], the Brauer and Manin obstruction is the only obstruction, for more information we refer to the Bourbaki exposé by Peyre [34].

By taking a linear combination of corestrictions

$$
\operatorname{cores}_{k_{v}(P) \mid k_{v}}: \operatorname{Br}\left(k_{v}(P)\right) \longrightarrow \operatorname{Br}\left(k_{v}\right),
$$

of evaluations $b(P)$ of $b \in \operatorname{Br}(X)$ at closed points $P$ of $X_{v}$, we define the evaluation of $b$ at local 0 -cycles. The Manin pairing is then extended to local 0-cycles, and it factorizes through the Chow groups of 0-cycles, cf., Colliot-Thélène [4].

$$
\prod_{v \in \Omega} \mathrm{CH}_{0}\left(X_{v}\right) \times \operatorname{Br}(X) \longrightarrow \mathbb{Q} / \mathbb{Z}
$$

Terminology. For a family of algebraic varieties, we say that the Brauer and Manin obstruction is the only obstruction to the Hasse principle for 0 -cycles of degree 1 if the existence of families of local 0 cycles of degree 1 orthogonal to $\operatorname{Br}(X)$ implies the existence of a global 0 -cycle of degree 1 .

We say that the Brauer and Manin obstruction is the only obstruction to weak approximation for 0-cycles of degree $\delta$, if the following statement is satisfied. Given a family of local 0-cycles $\left\{z_{v}\right\}_{v \in \Omega}$ of degree $\delta$ orthogonal to $\operatorname{Br}(X)$, for any positive integer $m$ and for any finite subset $S$ of $\Omega$, there exists a global 0 -cycle $z=z_{m, S}$ of degree $\delta$, such that $z$ and $z_{v}$ have the same image in $\mathrm{CH}_{0}\left(X_{v}\right) / m$, for all $v \in S$. 
It is conjectured that the Brauer and Manin obstruction explains the failure of the Hasse principle and weak approximation for 0-cycles on all proper smooth algebraic varieties, even though it is not the case for rational points. This is the main topic of this survey. We will state this main conjecture in a more elegant manner after reviewing the recent reformulation of the question presented in Section 2.

We remark that stronger obstructions exist for rational points, e.g., non-abelian descent obstructions or equivalently Brauer and Manin obstructions applied to étale covers, but they cannot be extended directly to 0-cycles.

2. Reformulation of the question and the main conjecture. Note that the Manin pairing for 0-cycles factorizes through the product of modified local Chow groups:

$$
\prod_{v \in \Omega} \mathrm{CH}_{0}^{\prime}\left(X_{v}\right) \times \operatorname{Br}(X) \longrightarrow \mathbb{Q} / \mathbb{Z}
$$

where

$\mathrm{CH}_{0}^{\prime}\left(X_{v}\right)= \begin{cases}\mathrm{CH}_{0}\left(X_{v}\right), & \text { if } v \text { is a non-archimedean place, } \\ \operatorname{Coker}\left(N_{\mathbb{C} \mid \mathbb{R}}: \mathrm{CH}_{0}\left(X_{\mathbb{C}}\right) \rightarrow \mathrm{CH}_{0}\left(X_{\mathbb{R}}\right)\right), & \text { if } v \text { is a real place, } \\ 0, & \text { if } v \text { is a complex place. }\end{cases}$

Given the complex

$$
\operatorname{Br}(k) \longrightarrow \bigoplus_{v \in \Omega} \operatorname{Br}\left(k_{v}\right) \longrightarrow \mathbb{Q} / \mathbb{Z},
$$

global 0-cycles are annihilated under the pairing, and the image of $\operatorname{Br}(k) \rightarrow \operatorname{Br}(X)$, paired with families of local 0-cycles of the same degree give 0 as well. We obtain a complex of local-global type

$$
\mathrm{CH}_{0}(X) \longrightarrow \prod_{v \in \Omega} \mathrm{CH}_{0}^{\prime}\left(X_{v}\right) \longrightarrow \operatorname{Hom}(\operatorname{Br}(X), \mathbb{Q} / \mathbb{Z}) .
$$

As the variety $X$ is supposed to be smooth, its Brauer group is torsion. One deduces the complex

(E) ${\underset{\lim }{n}}_{n} \mathrm{CH}_{0}(X) / n \longrightarrow \prod_{v \in \Omega}{\underset{\leftarrow}{n}}_{n} \mathrm{CH}_{0}^{\prime}\left(X_{v}\right) / n \longrightarrow \operatorname{Hom}(\operatorname{Br}(X), \mathbb{Q} / \mathbb{Z})$. 
Similarly, one can obtain a complex concerning 0 -cycles of degree 0 :

$$
\begin{aligned}
\lim _{n} A_{0}(X) / n \longrightarrow \prod_{v \in \Omega} & {\underset{\lim }{n}}_{n} A_{0}\left(X_{v}\right) / n \\
& \longrightarrow \operatorname{Hom}(\operatorname{Br}(X) / \operatorname{Br}(k), \mathbb{Q} / \mathbb{Z}),
\end{aligned}
$$

where $A_{0}(-)=\operatorname{Ker}\left[\operatorname{deg}: \mathrm{CH}_{0}(-) \rightarrow \mathbb{Z}\right.$ ], and by abuse of notation, we denote the cokernel Coker $(\operatorname{Br}(k) \rightarrow \operatorname{Br}(X))$ by $\operatorname{Br}(X) / \operatorname{Br}(k)$.

We remark that the exactness of $(\mathrm{E})$ and $\left(\mathrm{E}_{0}\right)$ is a birational invariant for proper smooth geometrically integral varieties defined over number fields. This is deduced from the birational invariance of Chow groups of 0-cycles [8, Proposition 6.3] and of Brauer groups [19, subsection 3.7].

The sequence (E) concerns both the Hasse principle and weak approximation, it also contains information of 0-cycles of any degree. Its exactness implies that

(i) the sequence $\left(\mathrm{E}_{0}\right)$ is exact [44, Remark 1.1 (ii)];

(ii) the Brauer and Manin obstruction is the only obstruction to the Hasse principle for 0-cycles of degree 1 [44, Remark 1.1 (iii)];

(iii) the Brauer and Manin obstruction is the only obstruction to weak approximation for 0-cycles of degree 1 [30, Proposition 2.2.1];

(iv) for any integer $\delta$, the Brauer and Manin obstruction is the only obstruction to weak approximation for 0-cycles of degree $\delta$, assuming the existence of a global 0-cycle of degree 1 [30, Proposition 2.2.1].

The reformulation above dates back to van Hamel [41]. Our main conjecture has been stated by several authors in various forms in the literature, cf., [4, Section 1], [5, Section 1], [11, Section 4], [26, Section 7].

Main conjecture. The sequence (E) is exact for all proper smooth geometrically integral algebraic varieties.

\section{Classical results for varieties of dimensions 0 and 1 .}

3.1. Dimension 0 . Let $k$ be a number field and let $X=\operatorname{Spec}(k)$, then the complex $(\mathrm{E})$ is exact for $X$. In fact, it is the Pontryagin dual 
of the exact sequence coming from the global class field theory

$$
\operatorname{Br}(k) \longrightarrow \bigoplus_{v \in \Omega} \operatorname{Br}\left(k_{v}\right) \longrightarrow \mathbb{Q} / \mathbb{Z} .
$$

This explains why one should modify local Chow groups at archimedean places in the sequence (E). This also shows the exactness of (E) for all $k$-rational varieties as well since, the degree map

$$
\operatorname{deg}: \mathrm{CH}_{0}\left(\mathbb{P}^{n}\right) \longrightarrow \mathrm{CH}_{0}(k)=\mathbb{Z}
$$

is an isomorphism.

3.2. Dimension 1 . Let $X$ be a smooth projective geometrically integral curve defined over a number field $k$. Its Jacobian $\mathrm{Jac}_{X}$ is an abelian variety defined over $k$. Assuming the conjectural finiteness of the Tate and Shafarevich group

$$
\amalg\left(k, \operatorname{Jac}_{X}\right)=\operatorname{Ker}\left[\mathrm{H}^{1}\left(k, \operatorname{Jac}_{X}\right) \longrightarrow \prod_{v \in \Omega} \mathrm{H}^{1}\left(k_{v}, \operatorname{Jac}_{X}\right)\right],
$$

the statement that the Brauer and Manin obstruction is the only obstruction to the Hasse principle for 0-cycles of degree 1 has been proved originally by Saito [36, Corollary 8.5]. A simplified alternative proof was given by Colliot-Thélène [5, Proposition 3.7]; moreover, a statement concerning weak approximation of 0-cycles of degree 1 was also proved in [5, Proposition 3.3]. Summarized by van Hamel, the exactness of (E) for curves was stated in [41].

If $X=E$ is an elliptic curve, then assuming the finiteness of $\amalg(k, E)$, the exact sequence $\left(\mathrm{E}_{0}\right)$ is part of the well-known Cassels and Tate dual exact sequence:

$$
\begin{aligned}
0 \longrightarrow \overline{E(k)} \longrightarrow \prod_{v \in \Omega} E\left(k_{v}\right)^{\prime} \longrightarrow \operatorname{Hom}\left(\mathrm{H}^{1}(k, E), \mathbb{Q} / \mathbb{Z}\right) \\
\longrightarrow \operatorname{Hom}(\amalg(k, E), \mathbb{Q} / \mathbb{Z}) \longrightarrow 0,
\end{aligned}
$$

where $E\left(k_{v}\right)^{\prime}$ is defined to be $E\left(k_{v}\right)$, for non-archimedean places, and otherwise the group of connected components $\pi_{0}\left(E\left(k_{v}\right)\right)$. Considering only the Hasse principle for 0 -cycles of degree 1 on curves, a sharper result was proved recently. Define the subquotient consisting of every- 
where locally constant elements in the Brauer group

$$
\mathrm{S}(X)=\operatorname{Ker}\left(\frac{\operatorname{Br}(X)}{\operatorname{Br}(k)} \longrightarrow \prod_{v \in \Omega} \frac{\operatorname{Br}\left(X_{v}\right)}{\operatorname{Br}\left(k_{v}\right)}\right) .
$$

The finiteness of $\amalg\left(k, \mathrm{Jac}_{X}\right)$ implies the finiteness of $\mathrm{B}(X)$. Eriksson and Scharaschkin [17] proved that the $\mathrm{B}(X)$-obstruction is the only obstruction to the Hasse principle for 0 -cycles of degree 1 on curves under the finiteness assumption of $\amalg\left(k, \mathrm{Jac}_{X}\right)$, i.e., the existence of local 0 -cycles of degree 1 orthogonal to $\mathrm{B}(X)$ implies the existence of a global 0-cycle of degree 1 .

In the next section, we summarize recent progress on the main conjecture.

\section{Recent progress on the conjecture for higher dimensional} varieties. Several families of higher dimensional varieties have been proved to satisfy the main conjecture, most of them are:

(i) (smooth compactifications of) homogeneous varieties;

(ii) fibrations over curves and projective spaces.

Moreover, a general relation has been found between the arithmetic (Brauer and Manin obstruction to the Hasse principle and to weak approximation) of rational points and the arithmetic of 0-cycles.

4.1. A relation between rational points and 0 -cycles. The following theorem permits us to deduce results on 0 -cycles from those on rational points.

Theorem 4.1. [30, Theorems A, B], [31, Theorem 2.1]. Let $L \mid k$ be a finite extension of number fields and let $\mathscr{K}_{L}$ be the set of finite extensions $K \mid k$ that are linearly disjoint from $L$.

Consider proper smooth geometrically rationally connected $k$-varieties $X$. Assume that the Brauer and Manin obstruction is the only obstruction to weak approximation (respectively to the Hasse principle) for $K$-rational points on $X_{K}$ for any $K \in \mathscr{K}_{L}$. Then, the sequence (E) is exact for $X$ (respectively the Brauer and Manin obstruction is the only obstruction to the Hasse principle for 0-cycles of degree 1 on X). 
It has been conjectured that the Brauer and Manin obstruction is the only obstruction to the Hasse principle and to weak approximation, for rational points on geometrically rationally connected varieties.

A similar result is available concerning only the Hasse principle for all proper smooth varieties.

Theorem 4.2. [30, Proposition 4.6.1]. Let $L \mid k$ be a finite extension of number fields and let $\mathscr{K}_{L}$ be the set of finite extensions $K \mid k$ that are linearly disjoint from $L$. Consider smooth proper $k$-varieties $X$ such that $\amalg\left(K, \underline{\operatorname{Pic}}_{X}^{0}\right)$ is finite for any $K \in \mathscr{K}_{L}$.

Assume that the $\mathrm{Б}\left(X_{K}\right)$-obstruction is the only obstruction to the Hasse principle for $K$-rational points on $X_{K}$ for any $K \in \mathscr{K}_{L}$. Then, the $\mathrm{E}(X)$-obstruction is the only obstruction to the Hasse principle for 0 -cycles of degree 1 on $X$.

4.2. Homogeneous varieties. Results for 0 -cycles on homogeneous varieties are all deduced via theorems in subsection 4.1 from known results for rational points. Until now, no direct argument for 0-cycles had been available. Theorem 4.3 is deduced from Theorem 4.1 and Borovoi's result for rational points [1, Theorem 2.3].

Theorem 4.3. Let $Y$ be a homogeneous space of a connected linear algebraic group $G$ defined over a number field, and let $X$ be a smooth compactification of $Y$. Suppose that the geometric stabilizer of $Y$ is connected (or abelian if $G$ is supposed semisimple simply connected). Then, the sequence (E) is exact for $X$.

In regards to the Hasse principle, Theorem 4.4 provides a sharper result for a broader range of homogeneous varieties. Theorem 4.4 is a consequence of Theorem 4.2, combined with the results on rational points in [1, Theorem 2.2], [2, Theorem 3.14] and [24, Theorem 1.1].

Theorem 4.4. Let $Y$ be a homogeneous space of a connected algebraic group $G$ defined over a number field $k$, and let $X$ be a smooth compactification of $Y$. Suppose one of the following hypotheses holds:

- $G$ is linear and the geometric stabilizer of $Y$ is connected; 
- $G$ is linear and semisimple simply connected, and the geometric stabilizer of $Y$ is abelian;

- $G$ is a semi-abelian variety (i.e., a commutative algebraic group which is an extension of an abelian variety by $\mathbb{G}_{m}^{r}$ ) and $Y$ is a principal homogeneous space, assuming the finiteness of the Tate and Shafarevich group of abelian varieties over number fields;

- the geometric stabilizer of $Y$ is connected and $k$ is totally imaginary, assuming the finiteness of the Tate and Shafarevich group of abelian varieties over number fields.

Then, the $\mathrm{B}(X)$-obstruction is the only obstruction to the Hasse principle for 0 -cycles of degree 1 on $X$.

4.3. Fibrations over projective spaces and curves. The exactness of (E) is known for projective spaces and curves. The fibration method allows one to deduce results for fibrations over such bases. However, lack of a suitable moving lemma for 0 -cycles on varieties other than curves prevents us from proving results for fibrations over more general bases.

4.3.1. Fibrations over projective spaces. Varieties considered here are often geometrically rationally connected, in most cases, similar results on rational points are also (sometimes conditionally) available.

Regarding the geometrical and arithmetical conditions supposed on fibres, two types of results have been established as follows:

(Type 1) Codimension 1 fibres are geometrically integral and the Brauer and Manin obstruction is the only obstruction to the Hasse principle and to weak approximation for almost all closed fibres;

(Type 2) Codimension 1 fibres are abelian-split and almost all closed fibres satisfy the Hasse principle and weak approximation.

Results of (Type 1) [28, Theorem A] are analogs of the work of Harari [20, 21, 22] for rational points on fibrations over projective spaces. It began with proving the result on fibrations over the projective line, followed by the induction argument which allows one to deal with the general higher dimensional cases. We require the notion of a generalized hilbertian subset which plays a key role in the proof that 
one can compare Brauer groups of the fibres over such subsets of closed points.

Definition 4.5. Let $V$ be an integral $k$-variety. A subset Hil of closed points of $V$ is called a generalized hilbertian subset of $V$, if there exist a finite étale morphism $\rho: Z \rightarrow U$ from an integral $k$-variety $Z$ onto a non-empty open subset $U$ of $V$ such that

$\mathrm{Hil}=\left\{\theta \in U ; \theta\right.$ is a closed point of $V$ and the fibre $\rho^{-1}(\theta)$ is connected $\}$.

Theorem 4.6. Let $X \rightarrow \mathbb{P}^{n}$ be a dominant proper morphism with integral geometric generic fibre $X_{\bar{\eta}}=X_{\eta} \otimes \overline{k\left(\mathbb{P}^{n}\right)}$ such that $\operatorname{Br}\left(X_{\bar{\eta}}\right)$ is finite and $\operatorname{Pic}\left(X_{\bar{\eta}}\right)$ is torsion-free (these are automatically satisfied if $X_{\bar{\eta}}$ is rationally connected). Suppose that:

(i) all 1-codimensional fibres are geometrically integral;

(ii) there exist a generalized hilbertian subset $\mathrm{Hil}$ of $\mathbb{P}^{n}$ such that the Brauer and Manin obstruction is the only obstruction to weak approximation (respectively the Hasse principle) for rational points or for 0 -cycles of degree 1 on the fibres $X_{\theta}$ for all $\theta \in \mathrm{Hil}$.

Then, the Brauer and Manin obstruction is the only obstruction to weak approximation (respectively to the Hasse principle) for 0-cycles of degree 1 on $X$. If $X_{\bar{\eta}}$ is furthermore supposed rationally connected, then $(\mathrm{E})$ is exact for $X$.

Results of (Type 2) date back to Salberger's devices for 0-cycles on conic bundle surfaces [37]. Parallel discussions for rational points and for 0-cycles on more general fibrations over the projective line have been provided in [15], and subsequently in [14], where Brauer elements coming from the function field of the base variety are used as key ingredients. The following result was initially announced (parallel with a statement for rational points) by Wittenberg in [43], and a complete proof was presented in [31] involving Theorem 4.6 with $n=1$ for the induction argument.

Theorem 4.7. Let $X \rightarrow \mathbb{P}^{n}$ be a dominant proper morphism with integral geometric generic fibre $X_{\bar{\eta}}=X_{\eta} \otimes \overline{k\left(\mathbb{P}^{n}\right)}$. Suppose that: 
- every 1-codimensional fibre $X_{\theta}$ contains an irreducible component $Y$ of multiplicity 1 , such that the algebraic closure of $k(\theta)$ in the function field $k(\theta)(Y)$ is an abelian extension of $k(\theta)$,

- there exist a generalized hilbertian subset $\mathrm{Hil}$ of $\mathbb{P}^{n}$ such that for all $\theta \in \mathrm{Hil}$ the fibres $X_{\theta}$ satisfy weak approximation (respectively the Hasse principle) for rational points or for 0-cycles of degree 1.

Then, the Brauer and Manin obstruction is the only obstruction to weak approximation (respectively the Hasse principle) for 0-cycles of degree 1 on $X$. If $X_{\bar{\eta}}$ is furthermore supposed rationally connected, then $(\mathrm{E})$ is exact for $X$.

For rational points, results similar to those of (Type 2) are conditional, and rely on Schinzel's hypothesis of rational prime numbers (this condition has been recently removed by analytic method in several cases, mostly over $k=\mathbb{Q}$ ). While in the argument for 0-cycles, Schinzel's hypothesis is replaced by Salberger's device.

Families of varieties defined by explicit equations (such as normic bundles over projective spaces) can also be dealt with in a similar manner. To define a (locally) closed subvariety of $R_{K \mid k} \mathbb{A}^{1} \times \mathbb{A}^{m}=\mathbb{A}^{d+m}$ naturally fibred over $\mathbb{A}^{m}$ via the parameters $\mathbf{t}$, consider affine equations of the form

$$
N_{K \mid k}(\mathbf{x})=P\left(t_{1}, \ldots, t_{m}\right),
$$

where $K \mid k$ is a finite extension of number fields of degree $d$ and where $P(\mathbf{t})$ is a non-zero polynomial (or a rational function) in $m$ variables $\mathbf{t}=t_{1}, \ldots, t_{m}$. Consider smooth compactifications of smooth loci of such varieties, they are (birationally) fibrations over $\mathbb{P}^{m}$. If $K \mid k$ is a cyclic extension, then the exactness of (E) has been assured by Theorem 4.7. The exactness of (E) for Châtelet surfaces are therefore included, it can also be deduced from Theorem 4.1 and the works $[12,13]$.

In the following cases, the exactness of (E) has been proved.

(i) $m=1$, the polynomial $P(t)=c t^{n_{1}}(1-t)^{n_{2}}$ with $c \in k^{*}$, follows from Theorem 4.1 as well as the results on rational points given in $[9,25,40]$; 
(ii) $m=1$, the extension $K \mid k$ is a Galois extension with

$$
\operatorname{Gal}(K \mid k)=\mathbb{Z} / n \mathbb{Z} \oplus \mathbb{Z} / n \mathbb{Z}
$$

The polynomial $P(t)$ is irreducible over $k$, and assuming $L=$ $k[t] /(P(t))$ contains a cyclic degree $n$ subfield of $K$, cf., [32, Corollary 2.3] and [42, Theorem 4.1], where the result was stated in a more general setting;

(iii) $m=1$, the extension $K \mid k$ is of degree 4 (not necessarily abelian), the polynomial $P(t)$ is of degree 2, irreducible over $k$ but split over $K$. This is deduced from Theorem 4.1, along with the results on rational points in [16, Theorem 1].

(iv) the extension $K \mid k$ is of prime degree (not necessarily cyclic), cf., [27, Example 3.1] and [42, Theorem 4.3];

(v) $K=k(\sqrt[n]{a})$ with $a \in k^{*}$ such that $a \notin k^{* q}$, for all integer $q$ dividing $n$, assuming that $n$ is coprime to $\varphi(n)$, where $\varphi(\cdot)$ is the Euler function. This is deduced by a restriction-corestriction argument [27, Example 3.3];

(vi) $K$ is the compositum of extensions $K_{1}, \ldots, K_{n}$ of distinct prime degrees, specifically if $K \mid k$ is a Galois extension of the degree of a product of two distinct primes. This is deduced from a restrictioncorestriction argument [27, Example 3.5, Remark 3.6];

(vii) the extension $K \mid k$ is a biquadratic extension, i.e., $\operatorname{Gal}(K \mid$ $k)=\mathbb{Z} / 2 \mathbb{Z} \oplus \mathbb{Z} / 2 \mathbb{Z}$, and the rational function $P(\mathbf{t})=Q(\mathbf{t})^{2}$ is a complete square in $k\left(t_{1}, \ldots, t_{m}\right)$, cf., [3];

4.3.2. Fibrations over curves. In general, the varieties considered here are no longer geometrically rationally connected. As mentioned in subsection 3.2, here we still assume the finiteness of the Tate and Shafarevich group $\amalg\left(k, \mathrm{Jac}_{C}\right)$ of the Jacobian of the base curve $C$. There are two related clues towards this direction. The first dates back to an argument by Colliot-Thélène for 0-cycles on fibrations over curves of arbitrary genus [6]. Subsequent progress was made by Frossard [18] on fibrations in Severi-Brauer varieties with the work by van Hamel [41] removing a restriction on the base field. Finally, in order to remove a subtle restriction on such fibrations, Wittenberg obtained a general result by reducing the question to fibrations over the projective line via the Weil restriction with respect to finite morphisms between curves [44]. 
The second clue began with three-folds constructed by Poonen [35] showing that the Brauer and Manin obstruction (even applied to étale covers) cannot explain the failure of the Hasse principle for rational points, these three-folds are fibrations over curves (of positive genus) in Châtelet surfaces. Though Poonen's three-folds cannot be covered by [44], Colliot-Thélène [7] showed that there exist global 0-cycles of degree 1, and the author showed in [29] the exactness of (E) on such three-folds. Combining the known arguments, the main result in [31] generalizes [29] and [44] at the same time. In summary, we state the following theorem.

Theorem 4.8. Let $X \rightarrow C$ be a dominant proper morphism to a smooth projective curve $C$ with geometrically rationally connected generic fibre. Assume the finiteness of the Tate and Shafarevich group $\amalg\left(k, \mathrm{Jac}_{C}\right)$. Suppose that:

(i) every closed fibre $X_{\theta}$ contains an irreducible component $Y$ of multiplicity 1 such that the algebraic closure of $k(\theta)$ in the function field $k(\theta)(Y)$ is an abelian extension of $k(\theta)$;

(ii) there exist a generalized hilbertian subset $\mathrm{Hil}$ of $C$ such that for all $\theta \in \mathrm{Hil}$ the fibre $X_{\theta}$ satisfies weak approximation (respectively the Hasse principle) for rational points or for 0 -cycles of degree 1.

Then, (E) is exact for $X$ (respectively the Brauer and Manin obstruction is the only obstruction to the Hasse principle for 0-cycles of degree 1 on $X)$.

\section{REFERENCES}

1. M. Borovoi, The Brauer and Manin obstructions for homogeneous spaces with connected or abelian stabilizer, J. reine angew. Math. 473 (1996), 181-194.

2. M. Borovoi, J.-L. Colliot-Thélène and A.N. Skorobogatov, The elementary obstruction and homogeneous spaces, Duke Math. J. 141 (2008), 321-364.

3. Y. Cao and Y. Liang, Local-global principle for certain biquadratic normic bundles, Acta Arith. 164 (2014), 137-144.

4. J.-L. Colliot-Thélène, L'arithmétique du groupe de Chow des zérocycles, J. Th. Bordeaux 7 (1995), 51-73.

5. , Conjectures de type local-global sur l'image de l'application cycle en cohomologie étale, in Algebraic K-theory, W. Raskind and C. Weibel, eds., Proc. Symp. Pure Math. 67, American Mathematical Society, Providence, 1999.

6. __ Principe local-global pour les zéro-cycles sur les surfaces réglées, J. Amer. Math. Soc. 13 (2000), 101-124. 
7. J.-L. Colliot-Thélène, Zéro-cycles de degré 1 sur les solides de Poonen, Bull. Soc. Math. France 138 (2010), 249-257.

8. J.-L. Colliot-Thélène and D. Coray, L'équivalence rationnelle sur les points fermés des surfaces rationnelles fibrées en coniques, Comp. Math. 39 (1979), 301332 .

9. J.-L. Colliot-Thélène, D. Harari and A.N. Skorobogatov, Valeurs d'un polynôme á une variable représentées par une norme, in Number theory and algebraic geometry, M. Reid and A.N. Skorobogatov, eds., Lond. Math. Soc. Lect. Notes 303 (2003), 69-89.

10. J.-L. Colliot-Thélène and J.-J. Sansuc, La descente sur une variété rationnelle définie sur un corps de nombres, C.R.A.S., Paris 284 (1977), 1215-1218.

11. , On the Chow groups of certain rational surfaces: A sequel to a paper of S. Bloch, Duke Math. J. 48 (1981), 421-447.

12. J.-L. Colliot-Thélène, J.-J. Sansuc and Sir Peter Swinnerton-Dyer, Intersections of two quadrics and Chatelet surfaces I, J. reine angew. Math. 373 (1987), 37-107.

13. Intersections of two quadrics and Chatelet surfaces II, J. reine angew. Math. 374 (1987), 72-168.

14. J.-L. Colliot-Thélène, A.N. Skorobogatov and Sir Peter Swinnerton-Dyer, Rational points and zero-cycles on fibred varieties: Schinzel's 10 Yongqi Liang hypothesis and Salberger's device, J. reine angew. Math. 495 (1998), 1-28.

15. J.-L. Colliot-Thélène and Sir Peter Swinnerton-Dyer, Hasse principle and weak approximation for pencils of Severi-Brauer and similar varieties, J. reine angew. Math. 453 (1994), 49-112.

16. U. Derenthal, A. Smeets and D. Wei, Universal torsors and values of quadratic polynomials represented by norms, Math. Ann. 361 (2015), 1021-1042.

17. D. Eriksson and V. Scharaschkin, On the Brauer and Manin obstruction for zero-cycles on curves, Acta Arith. 135 (2008), 99-110.

18. E. Frossard, Obstruction de Brauer and Manin pour les zéro-cycles sur des fibrations en variétés de Severi-Brauer, J. reine angew. Math. 557 (2003), 81-101.

19. A. Grothendieck, Le groupe de Brauer: I, II, III, Dix exposés sur la cohomologie des schéma, North-Holland, Amsterdam, 1968.

20. D. Harari, Méthode des fibrations et obstruction de Manin, Duke Math. J. 75 (1994), 221-260.

21. , Fléches de spécialisations en cohomologie étale et applications arithmétiques, Bull. Soc. Math. France 125 (1997), 143-166.

22. Spécialisation des conditions de Manin pour les variétés fibrées audessus de l'espace projectif, Comp. Math. 143 (2007), 603-617.

23. Y. Harpaz and A. Skorobogatov, Singular curves and the étale Brauer and Manin obstruction for surfaces, Ann. Sci. Ecole Norm. Sup. 47 (2014), 765-778.

24. D. Harari and T. Szamuely, Local-global principles for 1-motives, Duke Math. J. 143 (2008), 531-557. 
25. D.R. Heath-Brown and A. Skorobogatov, Rational solutions of certain equations involving norms, Acta Math. 189 (2002), 161-177.

26. K. Kato and S. Saito, Global class field theory of arithmetic schemes, Cont. Math. 55 (1986), 255-331.

27. Y. Liang, The local-global exact sequence for Chow groups of zerocycles, Math. Res. Lett. 22 (2015), 169-182.

28. Principe local-global pour les zéro-cycles sur certaines fibrations au-dessus de l'espace projectif, Bull. Soc. Math. France 142 (2014), 269-301.

29. , Principe local-global pour les zéro-cycles sur certaines fibrations au-dessus d'une courbe: I, Math. Ann. 353 (2012), 1377-1398.

30. Arithmetic of 0-cycles on varieties defined over number fields, Ann. Sci. Ecole Norm. Sup. 46 (2013), 35-56.

31. Astuce de Salberger et zéro-cycles sur certaines fibrations, Int. Math. Res. Not. 3 (2013), 665-692.

32. Towards the Brauer and Manin obstruction on varieties fibred over the projective line, J. Algebra 413 (2014), 50-71.

33. Yu.I. Manin, Le groupe de Brauer-Grothendieck en géométrie diophantienne, Act. Cong. Int. Math. 1 (1971), 401-411.

34. E. Peyre, Obstructions au principe de Hasse et á l'approximation faible, Astérisque 299 (2005), 165-193.

35. B. Poonen, Insuficiency of the Brauer and Manin obstruction applied to étale covers, Ann. Math. 171 (2010), 2157-2169.

36. S. Saito, Some observations on motivic cohomology of arithmetic schemes, Invent. Math. 98 (1989), 371-404.

37. P. Salberger, Zero-cycles on rational surfaces over number fields, Invent. Math. 91 (1988), 505-524.

38. A.N. Skorobogatov, Torsors and rational points, Cambridge University Press, Cambridge, 2001.

39. _ Beyond the Manin obstruction, Invent. Math. 135 (1999), 399-424.

40. M. Swarbrick Jones, A note on a theorem of Heath-Brown and Skorobogatov, Quart. J. Math. 64 (2013), 1239-1251.

41. J. van Hamel, The Brauer and Manin obstruction for zero-cycles on SeveriBrauer fibrations over curves, J. Lond. Math. Soc. 68 (2003), 317-337.

42. D. Wei, On the equation $N_{K / k}(\Xi)=P(t)$, Proc. Lond. Math. Soc. 109 (2014), 1402-1434.

43. O. Wittenberg, Intersections de deux quadriques et pinceaux de courbes de genre 1, Lect. Notes Math. 1901, Springer, New York, 2007. 
44. O. Wittenberg, Zéro-cycles sur les fibrations au-dessus d'une courbe de genre quelconque, Duke Math. J. 161 (2012), 2113-2166.

Institut de Mathématiques de Jussieu-Paris Rive Gauche, Université Paris Diderot, Paris 7, Bâtiment Sophie Germain, Cedex 13, 75013 Paris, France

Email address: yongqi.liang@imj-prg.fr 